\title{
Antithrombotic therapy after transcatheter aortic valve implantation: a new piece of the still unresolved puzzle
}

\author{
Giuseppe Gargiulo $^{1,2}$, Marco Valgimigli ${ }^{1}$ \\ ${ }^{1}$ Department of Cardiology, Bern University Hospital, Bern, Switzerland; ${ }^{2}$ Department of Advanced Biomedical Sciences, University Federico II of \\ Naples, Naples, Italy \\ Correspondence to: Marco Valgimigli, MD, PhD. Department of Cardiology, Bern University Hospital, CH-3010 Bern, Switzerland. \\ Email: marco.valgimigli@insel.ch. \\ Provenance: This is an invited Editorial commissioned by the Section Editor Hai-Long Dai (Department of Cardiology, Yan'an Affiliated Hospital of \\ Kunming Medical University, Kunming, China). \\ Comment on: Rodés-Cabau J, Masson JB, Welsh RC, et al. Aspirin Versus Aspirin Plus Clopidogrel as Antithrombotic Treatment Following \\ Transcatheter Aortic Valve Replacement With a Balloon-Expandable Valve: The ARTE (Aspirin Versus Aspirin + Clopidogrel Following \\ Transcatheter Aortic Valve Implantation) Randomized Clinical Trial. JACC Cardiovasc Interv 2017;10:1357-65.
}

Submitted Sep 28, 2017. Accepted for publication Oct 01, 2017.

doi: $10.21037 /$ jtd.2017.10.28

View this article at: http://dx.doi.org/10.21037/jtd.2017.10.28

The 2017 marks the $40^{\text {th }}$ anniversary of percutaneous coronary intervention (PCI), but also the $15^{\text {th }}$ anniversary for transcatheter aortic valve implantation (TAVI) (1). For more than 50 years, surgical aortic valve replacement (SAVR) has been the standard of care for patients with severe symptomatic aortic stenosis, improving outcomes and prolonging the lives of these patients. In 1990s the transcatheter delivery of a bioprosthetic aortic valve was conceptualized and largely tested in animals. The first human implantation of a percutaneous implantable prosthetic heart valve composed of 3 bovine pericardial leaflets mounted within a balloon-expandable stent was performed in Rouen on 16 April 2002 in a 57-year-old desperately ill man in cardiogenic shock, with critical aortic stenosis, subacute leg ischemia deemed inoperable due to multiple comorbidities (valve replacement had been declined for this patient, and balloon valvuloplasty had been performed with nonsustained results) (2).

Since then, TAVI has dramatically evolved, devices and procedural techniques have rapidly improved and results of randomized clinical trials have revolutionized the current treatment of severe aortic stenosis leading today to more than 300,000 procedures performed worldwide in more than 1,000 centers and 65 countries (1). Fifteen years after the first-in-man case, we can consider TAVI, with its explosive potential, as one of the major medical breakthroughs of the past decade in cardiology. Technological improvements and favorable clinical outcomes allowed to clearly establish the role of TAVI as the recommended strategy compared with medical therapy for inoperable patients, and alternative to surgery for high-risk patients, reaching more recently also the appropriate scientific evidence to recommend this procedure in patients at intermediaterisk (3-7). Furthermore, trials among low-risk patients are ongoing and evidence on feasibility and safety of TAVI in other clinical settings (treatment of patients with bicuspid aortic valve, pure native aortic regurgitation, degenerated surgical bioprosthetic valves or those with symptomatic moderate aortic stenosis or asymptomatic severe aortic stenosis) is being accumulated (3-7). However, ischemic and bleeding events soon after or at long-term after TAVI remain high, advocating dedicated investigations on optimal antithrombotic therapy (Figure 1) (8).

The prevention of ischemic complications during TAVI most likely requires full-dose anticoagulation. The most frequently used anticoagulant is unfractionated heparin, and the only trial comparing this latter with bivalirudin, the BRAVO 3 (Effect of Bivalirudin on Aortic Valve Intervention Outcomes 3), showed that bivalirudin did not reduce rates of major bleeding at 48 hours or net adverse cardiovascular events within 30 days compared with heparin (even if the non-inferiority hypothesis was reached for 


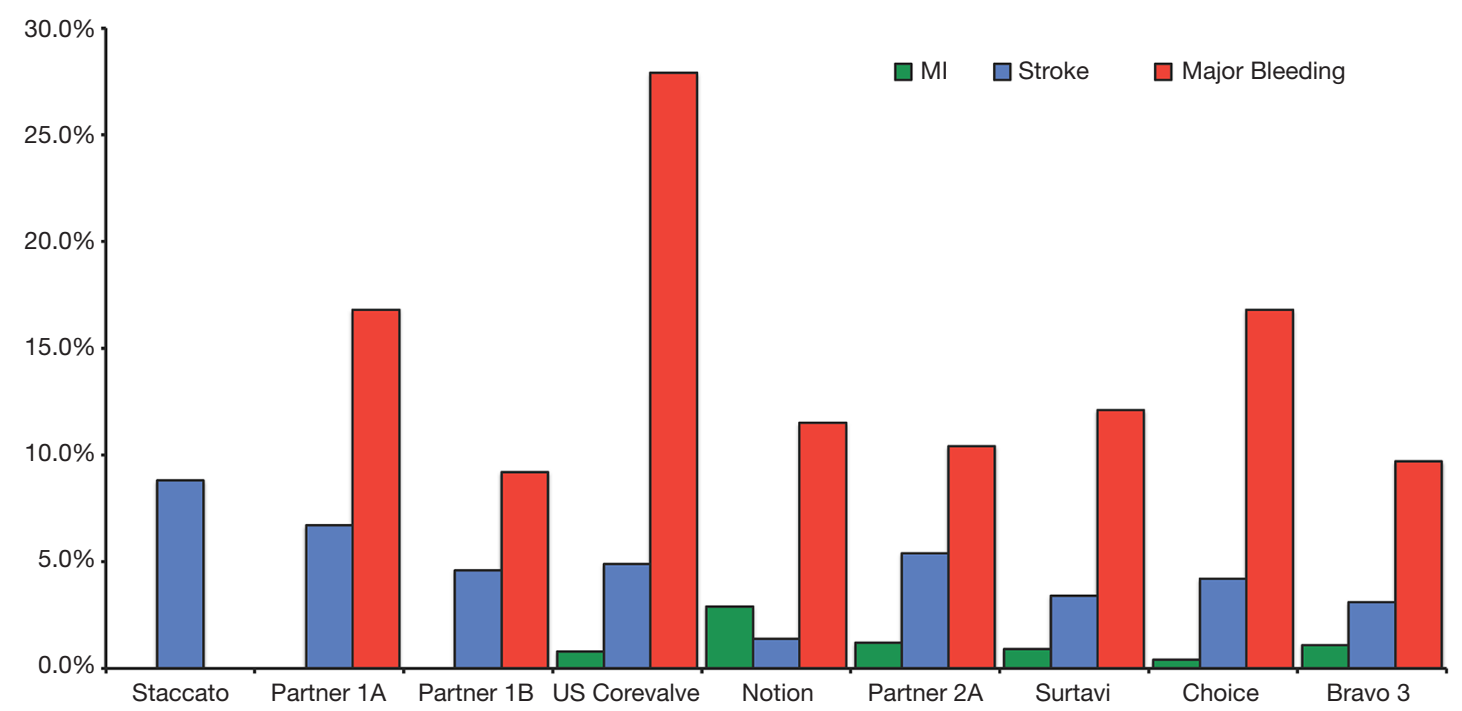

Figure 1 Rates of myocardial infarction, stroke and major bleeding 30 days after TAVI in main randomized clinical trials. Data on major bleeding were not available for STACCATO trial. The rate of myocardial infarction was $0 \%$ in STACCATO, PARTNER 1A and 1B. MI, myocardial infarction.

this endpoint) supporting the concept that heparin should remain the standard of care in TAVI patients (9). Regarding the post-procedural antithrombotic therapy, based on the increased thrombotic risks related to TAVI valve structure, dual antiplatelet therapy (DAPT) with aspirin (indefinitely) and clopidogrel (1 to 6 months) - in the absence of an indication for anticoagulation-has been a widely accepted empirical treatment, which was incorporated into practice guidelines. Differently from surgical interventions, the diseased native valve is not removed in TAVI procedures and may be mechanically damaged, leading to the exposure and/or embolism of valvular components into the arterial circulation. Moreover, the insertion of the prosthesis into the diseased native valve creates an irregular zone around the valve frame with modified flow patterns that may predispose to thrombus formation, particularly in the case of small valve sizes with associated patientprosthesis mismatch. Notably, TAVI patients remain at risk for stroke throughout the first months after the procedure and mechanisms other than valve manipulation seem to be involved (i.e., aortic wall injury, posttraumatic surface exposure with consequent activation of the hemostatic system, turbulence or local blood stasis, atrial fibrillation). For all these reasons, although empirical, the recommendation to adopt DAPT has reached widespread clinical practice. DAPT is a mainstay therapy for patients undergoing PCI compared with aspirin monotherapy, but the higher risks of bleeding and the well-known impact of bleeding on mortality represent a crucial aspect to be taken into account when deciding on its duration (10-16). In TAVI patients, two important considerations should be done: (I) TAVI patients are often old, frail and with multiple comorbidities leading to high risk of bleeding. Hence the balance between preventing ischemic complications at acceptable costs for bleeding remains a challenge in this population; (II) the benefits of DAPT over aspirin monotherapy have been questioned by recent evidence (17). Indeed, the evidence from 2 small randomized trials, some small observational studies and meta-analyses does not support the use of DAPT compared with aspirin alone due to increased risk of bleeding in the absence of sizable beneficial effects on ischemic endpoints (17).

Recently, a third randomized clinical trial has been published (18). This is a prospective, randomized, openlabel multicenter clinical trial including 222 patients undergoing TAVI with a balloon-expandable Edwards SAPIEN XT $(92.3 \%)$ or SAPIEN $3(7.7 \%)$ valves, with no indication to oral anticoagulation (OAC), who have been randomized to aspirin or DAPT. Overall, the patient population was old (mean age was 79 years) and the mean Society of Thoracic Surgeons score was 6.3. The investigators observed a significantly higher rate of major or life-threatening bleeding events in the DAPT group ( $10.8 \%$ vs. $3.6 \% ; \mathrm{P}=0.038)$. Notably, the excess of 
bleeding events occurred within 30 days, the majority of which was post-procedurally and mainly included vascular or access-site complications or gastrointestinal bleeding. This increased risk of bleeding was the major driver for the higher (despite statistically borderline: $15.3 \%$ vs. $7.2 \%$; $\mathrm{P}=0.065$ ) rate of the primary endpoint, consisting of death, myocardial infarction, transient ischemic attack/ischemic stroke, or major/life-threatening bleeding 3 months after TAVI. Individual ischemic endpoints and mortality did not different between the 2 regimens, although numerically higher in the DAPT group. These findings represent an important step forward in the field.

The study was prematurely stopped due to slow recruitment and lack of financial support. Overall 222 patients of the 300 planned were included. However, it is important to note that even the requirement of 300 patients only was established for exploratory purposes without a formal sample size assumption. Therefore, ARTE, as all previous studies, lacks sufficient study power and its findings should be interpreted with caution and considered hypothesis-generating only.

Even when pooling together the three available randomized trials available, DAPT does not offer ischemic benefits rather bleeding harms (19). Yet, the overall number of patients remains limited and uncertainty around the point estimates for ischemic endpoints remain considerable and do entail the possibility that DAPT may offer greater protection than aspirin monotherapy. Additionally, no data is currently available in patients receiving more contemporary TAVI devices (e.g., it is unknown if the additional skirt aimed at reducing post-implantation paravalvular leak may potentially increase thrombogenicity). The impact of the high on-treatment platelet reactivity associated with clopidogrel or aspirin (which seems more frequent in TAVI patients) is yet to be understood.

Five of the 16 severe bleeds observed in the ARTE trial were gastro-intestinal. Despite the claim of the investigators that proton-pump inhibitors (PPI) were not specifically recommended in patients included in the trial, one wonders if and how much the preventive use of PPI would have impacted that risk. The use of PPI is currently recommended in all DAPT patients, even if the risk of GI complications a prior is not high $(20,21)$.

Stroke mainly occurs in the acute phase after TAVI and cerebral protection devices have been proposed to integrate pharmacologic therapy to prevent such complications. Recent studies support benefits of embolic protection during TAVI, indirectly providing further evidence for the importance of embolic debris in periprocedural stroke events $(22,23)$.

While ARTE and the two prior trials investigated the concomitant use of clopidogrel in TAVI patients (18), there are arguments supporting the preferential use of OAC therapy instead of antiplatelet agents. First, there is still uncertainties regarding the exact mechanisms causing thrombotic events after TAVI and currently we cannot exclude that thrombin is the major driver or a co-driver of such complications. Second, the rates of pre-existing atrial fibrillation and new-onset atrial fibrillation are relevant in TAVI population and significantly impact on stroke and mortality $(24,25)$. Third, there is evidence that leaflet thrombosis of transcatheter heart valves could be a relatively novel and important mechanism of transcatheter heart valve-related thrombotic events or even valve failure. Data from two registries, the SAVORY (Subclinical Aortic Valve Bioprosthesis Thrombosis Assessed with Four-Dimensional Computed Tomography) and the RESOLVE (Assessment of Transcatheter and Surgical Aortic Bioprosthetic Valve Thrombosis and Its Treatment with Anticoagulation), suggest that subclinical leaflet thrombosis, represented by leaflet thickening on computed tomography, is more common with transcatheter valves compared with bioprosthetic surgical valves, and is associated with increased rates of stroke or TIA (26). Importantly, anticoagulation (both novel OACs and warfarin), but not DAPT, was effective in the prevention or treatment of subclinical leaflet thrombosis (26), providing the rationale for investigating OAC use in TAVI patients (27). However, the natural history and clinical significance of asymptomatic leaflet thrombosis remains unclear. Assuming OAC therapy to be the solution, does starting it earlier rather than later make any difference and for how long should it be prescribed? Considering the OAC-related bleeding risks, it seems logical for the time being, in the absence of compelling evidence that the decision on which antithrombotic therapy should be individualized, after with careful consideration of the risk/benefit profile.

Currently, the complex puzzle of the optimal antithrombotic therapy after TAVI remains intriguing but unsolved. Clinical trials are ongoing (Table 1) and their results will hopefully add the missing pieces in this complex puzzle of optimal antithrombotic therapy after TAVI.

\section{Acknowledgements}

None. 


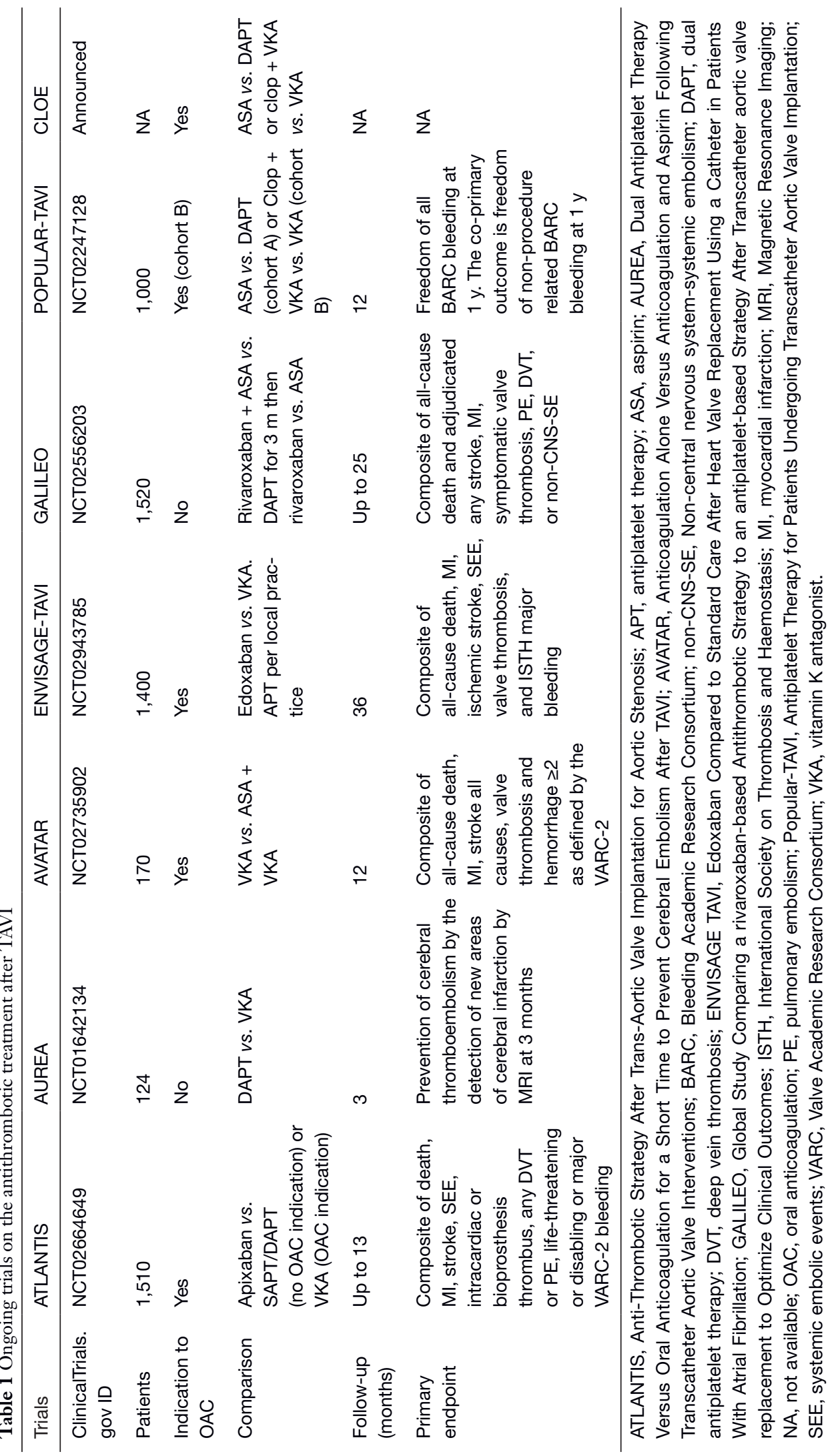




\section{Footnote}

Conflicts of Interest: The authors have no conflicts of interest to declare.

\section{References}

1. Cribier A. Commemorating the 15 -year anniversary of TAVI: insights into the early stages of development, from concept to human application, and perspectives. EuroIntervention 2017;13:29-37.

2. Cribier A, Eltchaninoff H, Bash A, et al. Percutaneous transcatheter implantation of an aortic valve prosthesis for calcific aortic stenosis: first human case description. Circulation 2002;106:3006-8.

3. Gargiulo G, Sannino A, Capodanno D, et al. Transcatheter Aortic Valve Implantation Versus Surgical Aortic Valve Replacement: A Systematic Review and Meta-analysis. Ann Intern Med 2016;165:334-44.

4. Baumgartner H, Falk V, Bax JJ, et al. 2017 ESC/EACTS Guidelines for the management of valvular heart disease: The Task Force for the Management of Valvular Heart Disease of the European Society of Cardiology (ESC) and the European Association for Cardio-Thoracic Surgery (EACTS). Eur Heart J 2017. [Epub ahead of print].

5. Leon MB, Smith CR, Mack MJ, et al. Transcatheter or Surgical Aortic-Valve Replacement in Intermediate-Risk Patients. N Engl J Med 2016;374:1609-20.

6. Reardon MJ, Van Mieghem NM, Popma JJ, et al. Surgical or Transcatheter Aortic-Valve Replacement in Intermediate-Risk Patients. N Engl J Med 2017;376:1321-31.

7. Thourani VH, Kodali S, Makkar RR, et al. Transcatheter aortic valve replacement versus surgical valve replacement in intermediate-risk patients: a propensity score analysis. Lancet 2016;387:2218-25.

8. Gargiulo G, Collet JP, Valgimigli M. Antithrombotic therapy in TAVI patients: changing concepts. EuroIntervention 2015;11 Suppl W:W92-5.

9. Dangas GD, Lefèvre T, Kupatt C, et al. Bivalirudin Versus Heparin Anticoagulation in Transcatheter Aortic Valve Replacement: The Randomized BRAVO-3 Trial. J Am Coll Cardiol 2015;66:2860-8.

10. Gargiulo G, Valgimigli M, Capodanno D, et al. State of the art: duration of dual antiplatelet therapy after percutaneous coronary intervention and coronary stent implantation - past, present and future perspectives. EuroIntervention 2017;13:717-33.
11. Gargiulo G, Windecker S, Vranckx P, et al. A Critical Appraisal of Aspirin in Secondary Prevention: Is Less More? Circulation 2016;134:1881-906.

12. Capodanno D, Gargiulo G, Buccheri S, et al. MetaAnalyses of Dual Antiplatelet Therapy Following DrugEluting Stent Implantation: Do Bleeding and Stent Thrombosis Weigh Similar on Mortality? J Am Coll Cardiol 2015;66:1639-40.

13. Valgimigli M, Costa F, Lokhnygina Y, et al. Trade-off of myocardial infarction vs. bleeding types on mortality after acute coronary syndrome: lessons from the Thrombin Receptor Antagonist for Clinical Event Reduction in Acute Coronary Syndrome (TRACER) randomized trial. Eur Heart J 2017;38:804-10.

14. Costa F, van Klaveren D, James S, et al. Derivation and validation of the predicting bleeding complications in patients undergoing stent implantation and subsequent dual antiplatelet therapy (PRECISE-DAPT) score: a pooled analysis of individual-patient datasets from clinical trials. Lancet 2017;389:1025-34.

15. Gargiulo G, Windecker S, da Costa BR, et al. Short term versus long term dual antiplatelet therapy after implantation of drug eluting stent in patients with or without diabetes: systematic review and meta-analysis of individual participant data from randomised trials. BMJ 2016;355:i5483.

16. Gargiulo G, Ariotti S, Santucci A, et al. Impact of Sex on 2-Year Clinical Outcomes in Patients Treated With 6-Month or 24-Month Dual-Antiplatelet Therapy Duration: A Pre-Specified Analysis From the PRODIGY Trial. JACC Cardiovasc Interv 2016;9:1780-9.

17. Hassell ME, Hildick-Smith D, Durand E, et al. Antiplatelet therapy following transcatheter aortic valve implantation. Heart 2015;101:1118-25.

18. Rodés-Cabau J, Masson JB, Welsh RC, et al. Aspirin Versus Aspirin Plus Clopidogrel as Antithrombotic Treatment Following Transcatheter Aortic Valve Replacement With a Balloon-Expandable Valve: The ARTE (Aspirin Versus Aspirin + Clopidogrel Following Transcatheter Aortic Valve Implantation) Randomized Clinical Trial. JACC Cardiovasc Interv 2017;10:1357-65.

19. Capodanno D, Angiolillo DJ. Antithrombotic Therapy for Prevention of Cerebral Thromboembolic Events After Transcatheter Aortic Valve Replacement: Evolving Paradigms and Ongoing Directions. JACC Cardiovasc Interv 2017;10:1366-9.

20. Valgimigli M, Bueno H, Byrne RA, et al.2017 ESC focused update on dual antiplatelet therapy in coronary artery 
disease developed in collaboration with EACTS: The Task Force for dual antiplatelet therapy in coronary artery disease of the European Society of Cardiology (ESC) and of the European Association for Cardio-Thoracic Surgery (EACTS). Eur Heart J 2017. [Epub ahead of print].

21. Gargiulo G, Costa F, Ariotti S, et al. Impact of proton pump inhibitors on clinical outcomes in patients treated with a 6- or 24-month dual-antiplatelet therapy duration: Insights from the PROlonging Dual-antiplatelet treatment after Grading stent-induced Intimal hyperplasia studY trial. Am Heart J 2016;174:95-102.

22. Haussig S, Mangner N, Dwyer MG, et al. Effect of a Cerebral Protection Device on Brain Lesions Following Transcatheter Aortic Valve Implantation in Patients With Severe Aortic Stenosis: The CLEAN-TAVI Randomized Clinical Trial. JAMA 2016;316:592-601.

23. Kapadia SR, Kodali S, Makkar R, et al. Protection Against Cerebral Embolism During Transcatheter Aortic Valve Replacement. J Am Coll Cardiol 2017;69:367-77.

Cite this article as: Gargiulo G, Valgimigli M. Antithrombotic therapy after transcatheter aortic valve implantation: a new piece of the still unresolved puzzle. J Thorac Dis 2017;9(11):42604265. doi: 10.21037/jtd.2017.10.28
24. Gargiulo G, Capodanno D, Sannino A, et al. New-onset atrial fibrillation and increased mortality after transcatheter aortic valve implantation: A causal or spurious association? Int J Cardiol 2016;203:264-6.

25. Sannino A, Gargiulo G, Schiattarella GG, et al. A metaanalysis of the impact of pre-existing and new-onset atrial fibrillation on clinical outcomes in patients undergoing transcatheter aortic valve implantation. EuroIntervention 2016;12:e1047-56.

26. Chakravarty T, Søndergaard L, Friedman J, et al. Subclinical leaflet thrombosis in surgical and transcatheter bioprosthetic aortic valves: an observational study. Lancet 2017;389:2383-92.

27. Windecker S, Tijssen J, Giustino G, et al. Trial design: Rivaroxaban for the prevention of major cardiovascular events after transcatheter aortic valve replacement: Rationale and design of the GALILEO study. Am Heart J 2017;184:81-7. 